\title{
Avaliação de protocolos para indução de inatividade ovariana em gatas domésticas
}

Débora Tramujas

BALLAROTTI $^{1}$

Wanderlei de MORAES 2

Cláudio Alvarenga de

OLIVEIRA $^{3}$

Érica C. FELIPPE ${ }^{3}$

Nei MOREIRA ${ }^{4}$

\section{Correspondência para:}

Débora Tramujas Balarotti, Rua General Canrobert Pereira da Costa, 1415 Centro - Toledo-PR, 85900-150 debora_ballarotti@hotmail.com

Recebido para publicação: 07/08/2008 Aprovado para publicação: 29/06/2009

\author{
1 - Programa de Pós-Graduação em Ciências Veterinárias da Universidade \\ Federal do Paraná, Curitiba-PR \\ 2- Criadouro de Animais Silvestres da Itaipu Binacional, Foz do Iguaçu-PR \\ 3 - Faculdade de Medicina Veterinária e Zootecnia da Universidade de São \\ Paulo, São Paulo-SP \\ 4 - Universidade Federal do Paraná, Palotina-PR
}

\section{Resumo}

Os índices de prenhez após inseminação artificial em felídeos selvagens não são satisfatórios devido ao variável ambiente endócrino após a estimulação com gonadotropinas. O objetivo deste estudo consistiu em aumentar a taxa de sucesso em programas de inseminação artificial em gatas domésticas (animal modelo). As fêmeas $(n=9)$ foram divididas em três grupos, cada um com três animais, sendo: 1) controle (C), somente $200 \mathrm{UI}$ eCG/ $100 \mathrm{UI}$ hCG ; 2) levonorgestrel oral (L) (0,075 mg) durante 37 dias + eCG/hCG; 3) etonogestrel (E), implante subdérmico durante 37 dias + eCG/hCG. Foram submetidas ao exame laparoscópico 29-39 horas após a administração de hCG para verificação da resposta ovariana e realização de esfregaço vaginal para monitoração da fase do ciclo estral. Foram coletadas amostras de fezes 60 dias antes e 60 dias após o tratamento com gonadotropinas para dosagem hormonal de estrógenos. Os resultados foram avaliados através do Teste ANOVA. Os níveis de significância mostraram que o Grupo E, em contraste com o Grupo C e o Grupo L, apresentou inibição satisfatória das concentrações de estrógenos durante a sua utilização. O grupo L não apresentou inibição ovariana durante o tratamento e diferença significativa em relação ao Grupo C. No exame laparoscópico todas as fêmeas dos grupos C, L e E apresentaram folículos e 77\% das fêmeas apresentaram corpo lúteo. Também apresentaram células epiteliais superficiais anucleadas e nucleadas características de estro. Concluiu-se que a utilização de implantes de etonogestrel em gatas domésticas mostrou-se eficaz, possibilitando a sua utilização prévia aos programas de inseminação artificial, aspiração folicular e também para a contracepção.

\section{Introdução}

As espécies de pequenos felídeos sul americanos apresentam baixo desempenho reprodutivo em cativeiro e estão sob alto risco de extinção na natureza. Considerando o alto valor genético da população de pequenos felídeos em cativeiro na América do Sul, e em razão de muitos indivíduos provenientes de vida livre serem de origem conhecida aumenta obrigatoriamente, a necessidade de um programa de conservação destas espécies. ${ }^{1}$
As técnicas de reprodução assistida utilizadas em felídeos consistem em coleta de sêmen pelo método de eletro-ejaculação, avaliação da função espermática, inseminação artificial, coleta e maturação de oócitos, fertilização e cultivo, transferência de embriões, formação de bancos de genoma e monitoração de hormônios através de técnicas não invasivas. ${ }^{1}$

A maioria dos estudos biológicos de felídeos tem focalizado o gato doméstico, que tornou-se um modelo intensivamente utilizado. $^{1}$

A meta deste estudo foi propiciar um 
melhor desempenho de gatas domésticas em programas de inseminação artificial com o controle sobre o ciclo reprodutivo. Tratamentos hormonais inéditos foram testados para a inativação ovariana temporária antes do protocolo para desenvolvimento folicular e indução da ovulação para a inseminação artificial. Sabese que a supressão do ciclo estral é indispensável para melhorar as taxas de fertilização em programas de inseminação artificial, em razão dos hormônios (secretados pelo ovário), interferirem com a terapia hormonal estabelecida. Portanto, neste estudo os esforços foram para desenvolver uma resposta hormonal mais uniforme, proporcionando um ambiente uterino mais adequado para a sobrevivência embrionária.

Este estudo integra um trabalho em conjunto com o Criadouro de Animais Silvestres da Itaipu Binacional (CASIB), o qual possibilita a futura aplicação dessas biotécnicas, desenvolvidas em gato doméstico, nas espécies ameaçadas de felídeos selvagens. O CASIB destaca-se nos cenários nacional e mundial na manutenção e reprodução de pequenos felídeos neotropicais, especialmente a jaguatirica, gato-do-mato-pequeno e gato-maracajá.

\section{Material e Método}

Foram estudadas nove fêmeas de gato doméstico, com idade entre 2 a 3,5 anos, consideradas sadias ao exame clínico, laboratorial (hemograma) e com histórico reprodutivo conhecido. Foram mantidas em gatis individuais e divididas em três grupos, cada um com três animais. Durante o estudo receberam ração comercial para gato doméstico e água ad libitum.

No grupo C (controle), as fêmeas foram tratadas com uma única dose intramuscular de 200 UI de eCG (gonadotropina coriônica eqüina, Novormon $500{ }^{\circledR}$, Syntex S.A/ Tecnopec, São Paulo-SP), para estimular o desenvolvimento folicular ovariano. Oitenta horas após, cada fêmea recebeu uma única injeção de 100 UI intramuscular de hCG (gonadotropina coriônica humana, Vetecor 5000 UI $^{\circledR}$, Laboratório Calier do Brasil LTDA, OsascoSP), para induzir a ovulação. Essas dosagens foram baseadas em experiências prévias, utilizando essas gonadotropinas em várias espécies felinas, incluindo o gato doméstico, sendo essas dosagens suficientes para induzir a foliculogênese e a ovulação. ${ }^{2}$

Os tratamentos de inibição ovariana foram estabelecidos para atingir a supressão ovariana por 37 dias, como o tempo médio equivalente à fase lútea de pseudoprenhez na gata doméstica. ${ }^{3}$

No grupo L, as fêmeas receberam levonorgestrel (progestágeno, Pilen ${ }^{\circledR}$, União Química, Embu-Guaçu - SP), via oral, o qual foi administrado na dose de $0,075 \mathrm{mg} /$ gata (1 comprimido de $0,75 \mathrm{mg}$ foi fracionado em dez partes), uma vez ao dia durante 37 dias, com o objetivo da indução da inatividade ovariana. Dois dias após o término da administração essas fêmeas receberam as mesmas doses anteriormente mencionadas de eCG e hCG.

No grupo E, as fêmeas receberam um implante contendo $68 \mathrm{mg}$ de etonogestrel (Implanon ${ }^{\circledR}$, Organon do Brasil Indústria e Comércio, São Paulo - SP), o qual permaneceu durante 37 dias, com o objetivo da indução da inatividade ovariana. Dois dias após a retirada do implante foram administrados eCG e hCG conforme protocolo já mencionado no grupo controle.

As fêmeas foram anestesiadas entre 29-39 h após a administração de hCG para o exame laparoscópico. Para a laparoscopia das fêmeas, um plano cirúrgico de anestesia foi induzido com xilazina $(0,5 \mathrm{mg} / \mathrm{kg}$, via intramuscular) (Calmium ${ }^{\circledR}$, Agener União, União Química, Embu-Guaçu - SP) e hidrocloridrato de cetamina $(15 \mathrm{mg} / \mathrm{kg}$, via intramuscular) (Ketamina ${ }^{\circledR}$, Agener União, União Química, Embu-Guaçu - SP) e mantido com anestesia inalatória com halotano (Fluothane ${ }^{\circledR}$, Astra Zeneca do Brasil, Cotia - SP).

Para a inserção e remoção dos implantes de etonogestrel, as fêmeas foram induzidas ao plano anestésico administrando- 
se hidrocloridrato de cetamina $(3-10 \mathrm{mg} /$ $\mathrm{kg}$ ) associada a xilazina $(0,5-1,5 \mathrm{mg} / \mathrm{kg})$, pela via intramuscular.

Após instituído plano anestésico, as fêmeas foram posicionadas em decúbito esternal. Uma incisão de $1,5 \mathrm{~cm}$ foi realizada na região cervical dorsal, cranial à escápula, para a inserção do implante. Os implantes foram removidos 37 dias após a sua inserção.

As gatas domésticas foram submetidas à laparoscopia para verificação da eficiência dos protocolos e resposta ovariana (presença de corpo lúteo, número e tamanho dos folículos). Todos os aspectos ovarianos e uterinos foram avaliados. A agulha de Verres (diâmetro de $2 \mathrm{~mm}$ ) foi utilizada para a manipulação das estruturas internas e mensuração precisa das estruturas anatômicas (ovariana e uterina). Os ovários foram visualizados diretamente através de um endoscópio rígido inserido na cavidade peritonial através de uma cânula colocada cranialmente à cicatriz umbilical. Após a laparoscopia, as fêmeas receberam uma injeção profilática subcutânea de amoxicilina $30 \mathrm{mg} / \mathrm{kg}$ em dose única.

Foram coletadas amostras fecais para dosagens de estrógenos, durante o período de 60 dias antes e durante o período de 60 dias após as laparoscopias. As amostras foram mantidas congeladas a $-20^{\circ} \mathrm{C}$ até a realização da análise. $\mathrm{O}$ processamento da amostra (extração e análise por RIE) foi realizado no Laboratório de Dosagens Hormonais (LDH) da Universidade de São Paulo (USP), seguindo o padrão já descrito por Brown et al. ${ }^{4}$. As amostras preparadas foram dosadas por meio de kits comerciais de radioimunoensaio em fase sólida, desenvolvidos para avaliação quantitativa de estradiol no soro humano, previamente validado para extratos de fezes de felinos. ${ }^{4}$

No momento da laparoscopia foram realizados esfregaços vaginais. Uma vez cranial ao orifício uretral, o swab foi friccionado contra a parede vaginal. As células foram então transferidas para uma lâmina de vidro através de rolamento gentil do swab. ${ }^{5}$ Os esfregaços foram fixados com spray fixador para cabelos e, após secas, as lâminas foram coradas com a técnica de Wright's-Giemsa (Diff-QuiK, Panótico) e observadas em microscópio óptico com aumento de $400 \mathrm{X}$ para caracterizar a morfologia das células epiteliais. Para cada exame, 100 células foram contadas em duplicata e classificadas com base na morfologia, coloração e tamanho do núcleo como: cornificada, intermediária ou parabasal. $^{5}$

Para avaliação do desempenho dos três grupos foi utilizado o método estatístico de comparação de médias ANOVA. A análise estatística foi dividida em duas etapas, nas quais os grupos que receberam tratamento para supressão ovariana (grupos $\mathrm{L}$, levonorgestrel e E, etonogestrel) foram comparados com o grupo C (controle) durante o período de tratamento para supressão ovariana (37 dias). Os grupos L e E foram também comparados com o grupo C durante e após o período de estimulação com as gonadotropinas exógenas.

Para verificar o ciclo estral de cada fêmea, foram quantificadas as concentrações de estrógenos fecais, sendo calculados os valores médios ( \pm erro padrão da média). Os valores basais de estradiol foram calculados utilizando um processo iterativo, no qual os valores que excederam a média + 2,0 DP (desvio padrão) foram excluídos. A média então foi recalculada e o processo de eliminação foi repetido até que nenhum valor excedesse a média + 2,0 DP. A média dos valores restantes foi considerada como valor basal.

\section{Resultados}

Durante a utilização do tratamento para supressão ovariana, o Grupo E apresentou diferença significativa $(p<0,05)$ nos níveis de estrógenos em relação aos Grupos C e L, observando diminuição significativa das concentrações de estradiol durante a utilização de etonogestrel. O grupo $\mathrm{L}$ não apresentou diferença significativa $(p>0,05)$ nos níveis de etrógenos em relação ao grupo $\mathrm{C}$. 
Durante a administração das gonadotropinas (eCG/hCG), os Grupos L e E (grupos que receberam tratamento hormonal para inibição ovariana prévia à administração de gonadotropinas) não apresentaram diferença significativa nos níveis de estrógenos em relação ao grupo $\mathrm{C}$ $(p>0,05)$, durante o período de estimulação ovariana.

Após o período de administração das gonadotropinas (eCG / hCG) os Grupos $\mathrm{L}$ e $\mathrm{E}$ (grupos que receberam tratamento hormonal para inibição ovariana prévia à administração de gonadotropinas) não apresentaram diferença significativa nos níveis de estrógenos em relação ao grupo $\mathrm{C}$ $(p>0,05)$, após o período de estimulação ovariana.

Os valores basais de estrógenos fecais do grupo $\mathrm{E}$ foram menores que os valores do grupo C e L. Verificou-se que os níveis de estrógenos durante o período de inibição ovariana do grupo $\mathrm{E}$ foram significativamente inferiores aos níveis de estrógenos dos grupos L e C. Após a administração das gonadotropinas não houve diferença significativa entre os grupos. Nas figuras 1 , 2 e 3 verifica-se os perfis da excreção fecal de estrógenos durante o período prévio à administração de gonadotropinas (eCG/ hCG), das gatas domésticas dos Grupos C, L e E, respectivamente.

Verificou-se que as gatas domésticas do grupo $\mathrm{E}$ apresentaram um maior número de folículos $(7,6 \pm 3,4)$ em relação às gatas domésticas dos grupos C $(6,3 \pm 1,4)$ e $\mathrm{L}$ $(4,0 \pm 0,57)$ (Figura 4). Com relação ao número de CLs, as gatas do grupo L tiveram maior número $(5,0 \pm 1,52)$ em relação ao grupo $\mathrm{C}(1,5 \pm 0,5)$ e $\mathrm{E}(2,0 \pm 0,57)$.

Verificou-se as fêmeas do Grupo C apresentaram $45 \%$ de células epiteliais cornificadas e $55 \%$ de células epiteliais intermediárias (Tabela 1). As fêmeas do grupo L apresentaram $72 \%$ de células epiteliais cornificadas e $28 \%$ de células

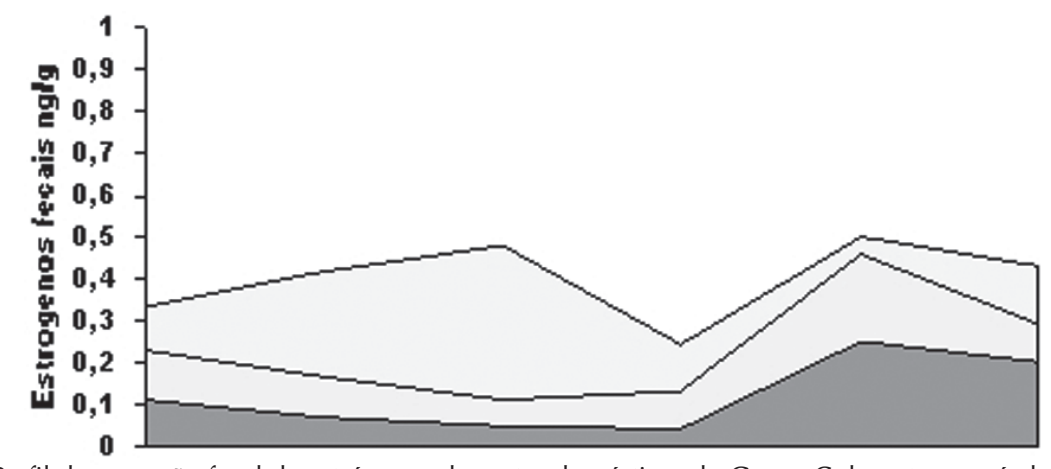

Figura 1 - Perfil da excreção fecal de estrógenos das gatas domésticas do Grupo C durante o período de inibição dos Grupos E e L

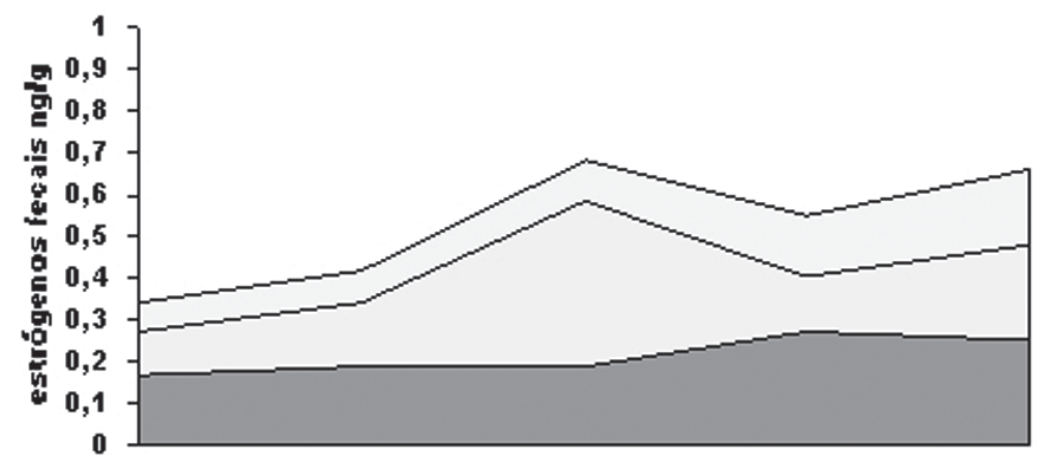

Figura 2 - Perfil da excreção fecal de estrógenos das gatas domésticas do Grupo L durante o período de inibição com o levonorgestrel oral $\mathbf{L}$ 


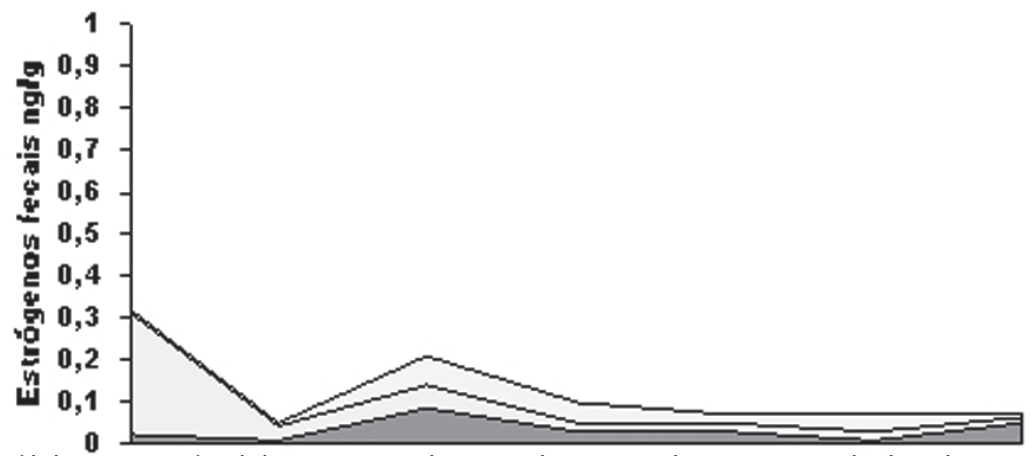

Figura 3 - Perfil da excreção fecal de estrógenos das gatas do grupo E durante o período de inibição com implante de etonogestrel $\mathbf{L}$

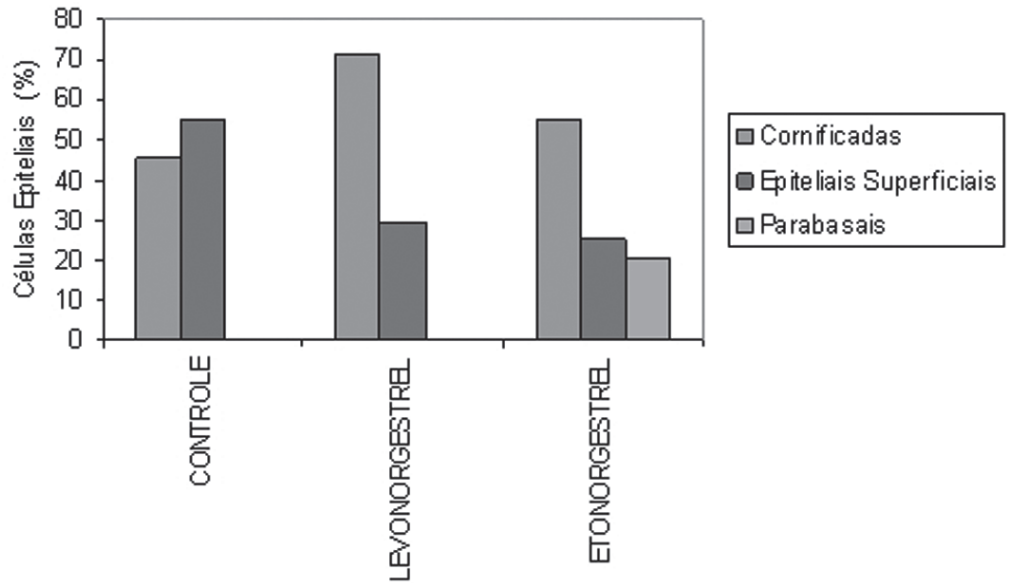

Figura 4 - Resultados da citologia vaginal realizada no momento do exame laparoscópico das gatas domésticas dos grupos C, L e E

Tabela 1 - Resultados do exame laparoscópico das gatas dos grupos etonogestrel, levonorgestrel e controle

\begin{tabular}{lccc}
\hline & CONTROLE & LEVONORGESTREL & ETONOGES TREL \\
\hline Diâmetro uterino (mm) & $7,0 \pm 0,31$ & $5,3 \pm 0,21$ & $5,5 \pm 0,34$ \\
Diâmetro ovariano (mm) & $9,0 \pm 0,83$ & $9,0 \pm 0,36$ & $6,4 \pm 0,20$ \\
Número de folículos / gata & $6,3 \pm 1,4$ & $4,0 \pm 0,57$ & $7,6 \pm 3,4$ \\
Diâmetro dos folículos (mm) & $1,68 \pm 1,13$ & $1,5 \pm 0,23$ & $1,34 \pm 0,08$ \\
Número de CLs/gata & $1,5 \pm 0,5$ & $5,0 \pm 1,52$ & $2,0 \pm 0,57$ \\
Diâmetro dos CLs (mm) & $2,5 \pm 0,5$ & $3,5 \pm 0,16$ & $2,16 \pm 0,3$ \\
\hline
\end{tabular}

epiteliais intermediárias. No grupo E as fêmeas apresentaram $45 \%$ de células intermediárias e $55 \%$ de células epiteliais cornificadas.

\section{Discussão}

Verificou-se que o implante de etonogestrel provocou supressão ovariana durante a sua utilização, indicando que a dose de $68 \mathrm{mg}$ /implante foi eficaz em gatas domésticas no estudo. Os resultados da análise estatística que compararam os níveis de estrógenos do grupo C, L e E, antes do período de estimulação com as gonadotropinas exógenas (eCG/hCG), mostraram que a inibição ovariana foi atingida nas gatas domésticas que receberam 
o implante de etonogestrel prévio ao protocolo de estimulação com as gonadotropinas exógenas (eCG/hCG). Foi verificado que o grupo $\mathrm{E}$ (etonogestrel) apresentou diferença significativa $(p<0,05)$ nos níveis de estrógenos em relação ao grupo C (controle) durante o período de inibição ovariana. Durante este período, as fêmeas do Grupo E apresentaram inatividade ovariana com baixas concentrações de estrógenos e ausência de picos. Segundo Mccann e Potter ${ }^{6}$, os contraceptivos apenas com progestágeno obtêm sua eficácia através do efeito inibidor da ovulação do progestágeno sobre o hipotálamo e hipófise. A supressão da secreção de gonadotropinas $(\mathrm{LH}$ e FSH) evita a ovulação. Conseqüentemente, o corpo lúteo está ausente e as concentrações de progesterona natural estão baixas. A inibição da ovulação pode ser determinada pela ausência de um pico de LH, supressão do desenvolvimento folicular e da produção de estradiol. De acordo com Davies et al. ${ }^{7}$, a ausência de ovulação e a alta eficácia contraceptiva assegurada por Implanon ${ }^{\circledR}$ (etonorgestrel) sugerem que este princípio ativo previne picos de LH.

Segundo Pelican et al. $^{8}$, estudos utilizando implantes de levonorgestrel em gatas domésticas indicaram que o mesmo inibe picos de estradiol e ovulação espontânea, apresentando resultados satisfatórios ao melhorar a resposta ovariana ao estímulo com gonadotropinas em programas de reprodução assistida. Em gatas domésticas, a supressão do ciclo estral através da utilização do hormônio levonorgestrel, prévio ao protocolo de inseminação artificial, melhora a resposta ovariana ao eCG e hCG. Ao contrário do resultado obtido por Pelican', a qual utilizou levonorgestrel na forma de implante, no presente estudo, o grupo L não apresentou diferença significativa $(p>0,05)$ em relação ao grupo $\mathrm{C}$ durante o período de inibição ovariana. Durante a administração do levonorgestrel oral, as fêmeas não apresentaram inibição ovariana, verificandose a presença de picos de estradiol em todas as fêmeas tratadas. Conseqüentemente o Grupo L não teve diferença em relação ao grupo C, não demonstrando a supressão ovariana desejada. Este resultado pode estar relacionado com o método de fracionamento do medicamento $\left(\right.$ Pilen $^{\circledR}$ ) e a dose utilizada, o que pode ter influenciado de forma negativa nos resultados observados. Novos estudos podem ser realizados utilizando doses diferentes, ou na mesma dose já utilizada com a manipulação do hormônio propriamente dito. Levandose em consideração a relação custo/benefício, a utilização de levonorgestrel oral em novos estudos deve ser investigada devido ao menor custo e facilidade de administração, podendo ser adicionado ao alimento.

As fêmeas dos grupos E e L não apresentaram diferença significativa ( $p>0,05)$ nos níveis de estrógenos em relação ao grupo C durante e após a administração de gonadotropinas exógenas. Segundo Pelican?, os estímulos com gonadotropinas aumentam o tempo de duração do estro e das concentrações de estradiol. Sabe-se que a estimulação com as gonadotropinas induz a uma segunda onda folicular em gatas, levando à prolongada exposição aos estrógenos. De acordo com a análise fecal, durante o período de estimulação com gonadotropinas, duas das três fêmeas do grupo E estavam com concentrações basais de estradiol, e no momento do exame laparoscópico, todas as fêmeas deste grupo apresentaram picos de estradiol. Após a laparoscopia apresentaram diminuição das concentrações de estradiol. Verifica-se desta forma, que as gatas deste grupo (Grupo E) apresentaram uma resposta uniforme e constante dos ovários à estimulação com as gonadotropinas exógenas, não havendo interferência de gonadotropinas endógenas na resposta ovariana. A ausência de um aumento excessivo nas concentrações de estradiol após a laparoscopia é benéfica, não ocorrendo o hiperestrogenismo, o qual alteraria o ambiente uterino e diminuiria a taxa de prenhez. Nenhuma das fêmeas do grupo L apresentou picos de estradiol no momento da laparoscopia. Duas das três 
fêmeas deste grupo apresentaram concentrações de estradiol aumentadas no período da estimulação ovariana com as gonadotropinas exógenas (antes da laparoscopia). Após a laparoscopia, duas das três fêmeas do grupo L apresentaram concentrações de estradiol superiores às encontradas no momento da laparoscopia. Estas concentrações de estradiol permaneceram aumentadas por aproximadamente 4 dias. Apenas uma, das três fêmeas deste grupo, não apresentou picos de estradiol durante e após a estimulação com as gonadotropinas. Dessa forma, verifica-se uma resposta não uniforme das fêmeas à estimulação, ocorrendo hiperestrogenismo após a laparoscopia em duas das três fêmeas tratadas com levonorgestrel oral. No grupo C foi verificado que todas as fêmeas apresentaram aumento das concentrações de estradiol no momento do exame laparoscópico e que uma das fêmeas deste grupo apresentou concentrações basais de estradiol no momento da estimulação com as gonadotropinas. Em uma das três fêmeas, as concentrações de estradiol após a laparoscopia ( \pm 5 dias) foram superiores às concentrações no momento da laparoscopia. As concentrações de estradiol permaneceram elevadas por \pm 9,3 dias, ocorrendo hiperestrogenismo nesta fêmea. Alguns fatores que resultam na diminuição das taxas de fertilização e de nidação embrionária em felídeos são as elevadas e prolongadas concentrações de estradiol, elevada secreção de progesterona ou luteólise prematura, as quais são ocasionadas pela hiperestimulação ovariana. Outro contribuinte para a baixa eficiência reprodutiva seguida de estimulação com gonadotropinas em felídeos selvagens é o desenvolvimento de uma segunda onda folicular e ovulação após 5-7 dias da primeira onda folicular. O desenvolvimento do folículo acessório e desenvolvimento luteal resultam numa elevada e prolongada concentração de estradiol e progesterona, os quais estão associados à interferência no transporte do embrião pelo oviduto e diminuição das taxas de prenhez.'
Neste estudo, todas as fêmeas do grupo L e duas das três fêmeas do grupo $\mathrm{E}$ apresentaram picos de estradiol fora do período de tratamento (inibição e estimulação com gonadotropinas). Verificase então que cinco das nove fêmeas do estudo apresentaram picos de estradiol em períodos que não foram administrados hormônios exógenos (antes do período de supressão ovariana e após o período de estimulação com gonadotropinas). As concentrações de estradiol dos picos das fêmeas que responderam ao estímulo com gonadotropinas foram maiores que as concentrações de estradiol de outros picos apresentados fora do período dos tratamentos (inibição ovariana e gonadotropinas exógenas).

No exame laparoscópico realizado 29-39 h após a administração de hCG, foi observado que as fêmeas do grupo C (Controle, $n=3$ ) apresentaram uma média de 6,3 folículos/gata, totalizando 19 folículos. Apenas duas das três fêmeas deste grupo apresentaram CL, uma com um e outra com dois CLs. As fêmeas do grupo L (Levonorgestrel, $\mathrm{n}=3$ ) apresentaram em média 4,0 folículos/gata, totalizando 12 folículos. Todas as fêmeas deste grupo apresentaram CL, em média 5,0 CLs/gata, totalizando 15 CLs. As fêmeas do grupo E (Etonogestrel, $\mathrm{n}=3$ ), apresentaram em média 7,6 folículos/gata, totalizando 23 folículos. Com relação à presença de CL, duas das três fêmeas apresentaram 2,0 CLs/gata, totalizando 4 CLs. Segundo Pelican9 ovários com múltiplos e recentes pontos de ovulação geralmente resultam nos melhores índices de prenhez em gatas domésticas. Recentes pesquisas em felídeos indicaram que fêmeas com a presença de CL maduro no momento da IA não atingem a prenhez. No grupo $\mathrm{L}$ (Levonorgestrel) foi observado um maior número de CLs/gata doméstica no momento do exame laparoscópico, o que teoricamente deveria resultar em uma maior fertilidade, no caso dessa espécie que é multípara (número de 4 a 5 filhotes)..$^{10} \mathrm{Em}$ fêmeas de pequenos felídeos silvestres sulamericanos, como por exemplo, o gato- 
do-mato-pequeno e o gato-maracajá, que normalmente têm apenas um a dois filhotes, espera-se como situação ideal um número menor de CLs recentes (como um a três), o que seria fisiológico para essas espécies. Um número excessivo de ovulações nessas espécies de felídeos silvestres pode estar associado a casos de hiper-estimulação ovariana, seguida de hiperestrogenismo e poliovulação, o que pode levar à produção de um número excessivo de filhotes, com menor peso e menor taxa de sobrevivência pós-parto. ${ }^{11}$ Resumindo, busca-se com esses protocolos hormonais, a resposta mais fisiológica possível, para os procedimentos de inseminação artificial. No caso de procedimentos de aspiração folicular para produção in vitro (PIV) de embriões, busca-se uma resposta ovariana com produção de maior número de folículos.

Ao exame microscópico de citologia vaginal foi observado que todas as fêmeas do estudo apresentaram células epiteliais vaginais características de estro. No grupo C, 45,3\% das células epiteliais eram superficiais anucleadas e $54,7 \%$ das células eram superficiais nucleadas. No grupo L, $71 \%$ das células observadas eram células superficiais anucleadas e $29 \%$ eram células superficiais nucleadas. Isto indica que nesses dois grupos as fêmeas estavam em estro de período avançado. Já no grupo E, houve a presença de células intermediárias (20\%), o que segundo Thrall e Olson ${ }^{5}$, no período de estro as células intermediárias estão presentes em pequeno número e as células epiteliais vaginais tornam-se progressivamente cornificadas quando as concentrações de estradiol aumentam. Neste grupo $54,7 \%$ das células eram epiteliais superficiais anucleadas (cornificadas) e $75 \%$ das células eram superficiais nucleadas, classificando-se como início de estro nas fêmeas deste grupo no momento da laparoscopia.

\section{Conclusão}

A utilização do implante de etonogestrel (68 mg/implante/37 dias) prévia à administração de gonadotropinas exógenas em gatas domésticas induziu inatividade ovariana, mostrando-se eficaz, sugerindo a possibilidade de utilização prévia em programas de inseminação artificial em felídeos selvagens.

O progestágeno levonorgestrel oral, na dose de $0,075 \mathrm{mg} /$ dia durante 37 dias/gata, não provocou supressão ovariana durante a sua utilização no estudo.

A inibição da atividade ovariana obtida pelo uso do etonogestrel em gata doméstica possibilita sua utilização como contraceptivo.

\section{Agradecimentos}

À Universidade Federal do Paraná Campus Palotina - Prof. Nei Moreira, agradeço pelo apoio, o qual foi meu orientador durante o mestrado. Ao CASIB (Criadouro de Animais Silvestres da Itaipu Binacional) Wanderlei de Moraes, agradeço pelo apoio e colaboração no projeto. À Universidade de São Paulo - LDH (Laboratório de Análises Hormonais) - Prof. Dr. Cláudio Alvarenga de Oliveira e Técnica Érica Felippe pelo apoio e colaboração para a realização das análises laboratoriais do projeto.

\section{Abstract}

Reproductive success in endangered captive small felids species is very low. Due to great variability in endocrine environment post gonadotropin treatment, after artificial insemination pregnancy rates are very low. Nowadays, ovarian activity controll improves the AI success in many species. In this study, new protocols were compared to improve the fertilization rates in artificial insemination programs in domestic cat. Female domestic cats were divided in three treatments: 1) control (eCG/hCG); 2) levonorgestrel $(0.075 \mathrm{mg})$ orally during 37 days + eCG/hCG; 3) etonogestrel subdermal implant during 37 days + eCG/hCG: Laparoscopies were done 29-39 hours post hCG
Key words: Artificial insemination. Ovary.

Wild felids. Contraception. Feline. Reproduction. 
treatment to verify ovarian activity. Vaginal swabs were collected at laparoscopic procedures. Fecal samples were colected 60 days before, during and 60 days after the gonadotropin treatment for estradiol assay. Means comparisons were done by ANOVA test. Results demonstrated that etonogestrel (implant) and not oral levonorgestrel successfully suppressed ovarian activity. The levonorgestrel group did not show ovarian inactivity during the administration, presenting oestradiol peaks and without significative diference comparing to control group. All females presented anuclear and nuclear superficial vaginal epithelial cells at laparoscopies. In conclusion, the etonogestrel implant used in the domestic cat was efficient and can be used previous to gonadotropin protocol in artificial insemination programs, follicular aspiration and contraception.

\section{Referências}

1 SWANSON, W. F; WILDT, D. E. Strategies and progress in reproductive involving small cat species, Review Small Felids Reproduction Research. International Zoo Yearbook, v. 35, n. 1, p. 152-159, 1997.

2 HOWARD, J. G.; DONOGHUE, A. M.; BARONE, M. A.; GOODROWE, K. L.; BLUMER, E. S. SNODGRASS, K.; STARNES, D.; TUCKER, M.; BUSH, M.; WILDT, D. E. Successful induction of ovarian activity and laparoscopic intrauterine artificial insemination in the cheetah (Acinoyix jubatus). J Zoo Wild Med, v. 23 p. 288-300, 1992.

3 WILDT, D. E.; BROWN, J. L.; SWANSON, W. F. Cats. In: KNOBIL, E.; NEIL, J. D. (Ed.). Encyclopedia of reproduction. New York: Academic Press, 1998. v. 1, p. $497-510$

4 BROWN, J. L.; WASSER, S. K.; WILDT, D. E.; GRAHAM, L. H. Comparative aspects of steroid hormone metabolism and ovarian activity in felids, measured noninvasively in feces. Biology of Reprodution, v. 51, p. 776-786, 1995.

5 THRALL, M. A.; OLSON, P. N. The vagina. In: THRALL, M. A.; OLSON, P. N. Diagnostic cytology and hematology of the dog and cat. St Louis: Mosby, 1999. p. 240-248.

6 MCCANN, M. F.; POTTER, L. S. Progestin-only contraception: a comprehensive review. Contraception, v. 50, p. S1-198, 1994. Supplement 1.

7 DAVIES, G. C.; FENG, L. X.; NEWTON, J. R.; VAN BEEK A.; COELINGH- BENNINK, H. J. Release characteristics, ovarian activity and menstrual bleeding pattern with a single contraceptive implant releasing 3-ketodesogestrel. Contraception, v. 47, p. 251-261, 1993.

8 PELICAN, K. M.; WILDT, D. E.; OTTINGER, M. A.; HOWARD, J. G. Short-term ovarian suppression with levonorgestrel before gonadotropin stimulation improves ovarian response for IVF in the domestic cat. Theriogenology, v. 57, p. 679, 2002.

9 PELICAN, K. M. Ovarian suppression with the progestin levonorgestrel but not the $\mathrm{GnRH}$ antagonis antide, induces a consistent response to exogenous gonadotropin in the domestic cat. Tesis (Doctor) National Zoological Park, Smithsonian Institution, Washington, DC, 2004.

10 JOHONSON, C. A. Distúrbios do sistema reprodutivo In: NELSON, R. W; COUTO,C. G. Medicina interna de pequenos animais. 3. ed. [S.I] Elsevier, 2006. p. 851.

11 WILDT, D. E.; SWANSON, W. .F.; BYERS, A. P.; ROTH, T. L., HOWARD, J. G. Sensitivity to exogenous gonadotropins for ovulation induction and laparoscopic artificial insemination in the Cheetah and clouded leopard. Biologic of Reproduction, v. 56, p. 10591068, 1997. 